\title{
The compact polarised emission of AGN - do many AGN jets have helical magnetic fields?
}

\author{
Denise C. Gabuzda* \\ Department of Physics, University College Cork, Republic of Ireland \\ E-mail: gabuzda@phys.ucc.ie
}

\begin{abstract}
To impose some kind of order on a broad review of various recent observational VLBI polarisation results, these results will be considered in the framework of the hypothesis that many Active Galactic Nuclei (AGN) jets have helical magnetic (B) fields. The results of the MOJAVE project, in which 133 radio-bright AGN are regularly monitored at $15 \mathrm{GHz}$ with polarisation sensitivity with the VLBA, represents an extremely useful resource that is now available to the astronomical community. There are a growing number of AGN whose jets show clear evidence for the presence of helical $\mathbf{B}$ fields: extended regions in the VLBI jets where the predominant $\mathbf{B}$ field is orthogonal to the jet direction, stretches of VLBI jets where the inferred $\mathbf{B}$ field remains nearly orthogonal to the jet direction in the presence of substantial bending of the jet, the common occurrence of "spine+sheath" jet polarisation structures and the presence of Faraday-rotation gradients across the VLBI jets. This compels us to take very seriously the idea that some, possibly many, AGN jets have helical $\mathbf{B}$ fields. In fact, such fields can come about very naturally as a consequence of the joint action of rotation of the central black hole+accretion disc and the jet outflow. As a whole, the recent observational results considered here suggest that we must look at AGN jets as fundamentally electromagnetic, current-carrying structures if we wish to fully understand their nature. Possible observational tests to distinguish between alternative interpretations for some of the observed phenomena are also discussed.
\end{abstract}

The 8th European VLBI Network Symposium

September 26-29, 2006

Toruń, Poland

${ }^{*}$ Speaker. 


\section{Introduction}

Most or all of the radio emission of Active Galactic Nuclei (AGN) is associated with jets of plasma that emerge from their central regions, presumably along the rotational axis of a central supermassive black hole that provides the ultimate source of energy for the nuclear activity. These jets are present on the smallest scales that can be probed with Very Long Baseline Interferometry (VLBI), and sometimes extend out to scales of many kiloparsecs. The observed radio emission is synchrotron radiation generated by relativistic electrons moving through regions with magnetic (B) fields and accelerated by these fields. The synchrotron radiation given off by each individual electron is highly concentrated in the direction of motion of the electron, and the radiation observed for an ensemble of relativistic electrons will, in general, be linear polarised if the synchrotron $\mathbf{B}$ field is at least partially ordered. Linear-polarisation observations can provide direct information about the degree of order and orientation of the $\mathbf{B}$ field giving rise to the observed synchrotron radiation.

The degree of polarisation of synchrotron radiation will depend to some extent on the spectrum (spectral index $\alpha, S \propto v^{\alpha}$ ) of the radiating region (Pacholczyk 1970). In the optically thin regime, when most emitted synchrotron photons will escape from the source without being absorbed, the maximum degree of linear polarisation is approximately $75 \%$ if the $\mathbf{B}$ field is perfectly ordered and the pitch-angle distribution for the electrons is random; the plane of linear polarisation is orthogonal to the associated $\mathbf{B}$ field. In the optically thick regime, when an emitted synchrotron photon will typically be absorbed and re-emitted many times on its way out of the source, the maximum degree of linear polarisation is approximately $10 \%$, and the plane of linear polarisation is parallel to the associated $\mathbf{B}$ field. In both the optically thin and thick cases, the observed degree of polarisation will decrease with increasing inhomogeneity of the $\mathbf{B}$ field. The expected $90^{\circ}$ rotation in the orientation of the observed polarisation angle in the transition between the optically thin and thick regimes has been observed in a number of cases (e.g. OJ287: Gabuzda \& Gómez 2001; 1308+326: Bezrukovs \& Gabuzda, these proceedings).

The observed radio polarisation angles can be affected by Faraday rotation - a rotation of the plane of polarisation that will occur if a linearly polarised electromagnetic wave travels through a region with free electrons and a $\mathbf{B}$ field with a non-zero component along the line of sight toward the observer. The origin of this rotation is that, under these conditions, the right-circularly-polarised (RCP) and left-circularly-polarised (LCP) components of the electromagnetic wave will have different indices of refraction, and so different propagation speeds through the magnetised plasma. The rotation of the polarisation angle $\chi$ is determined by the square of the observing wavelength $\lambda$, and the integral of the density of free electrons $N(s)$ and the line-of-sight component of the $\mathbf{B}$ field in the plasma (e.g. Burn 1966):

$$
\begin{aligned}
& \chi=\chi_{0}+\frac{e^{3} \lambda^{2}}{8 \pi m_{e}^{2} \varepsilon_{0} c} \int N(s) \vec{B}(s) \cdot d s \\
& \chi=\chi_{0}+\operatorname{RM} \lambda^{2}
\end{aligned}
$$

where $\chi_{0}$ is the intrinsic polarisation angle, $e$ is the electron charge, $m_{e}$ is the electron mass, and the integral is taken over the line of sight from the source to the observer. The coefficient of $\lambda^{2}$ is called 

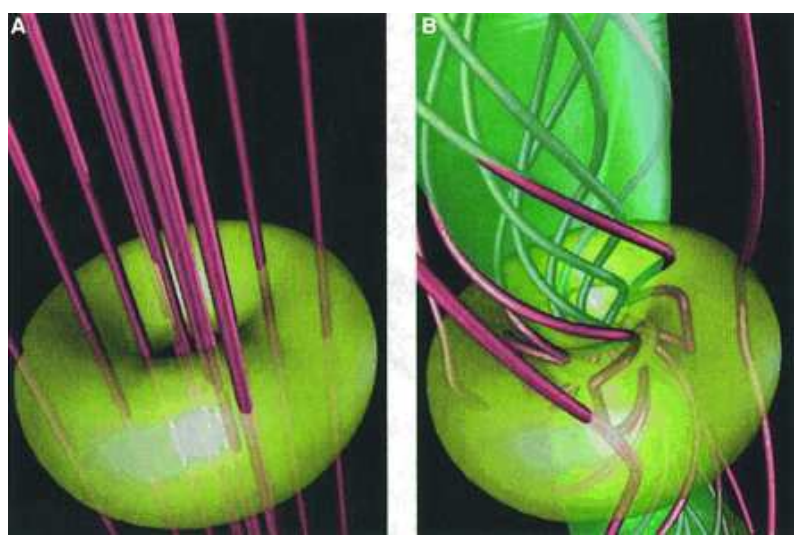

Figure 1: Figure 5 of Meier, Koide \& Uchida (2001), which illustrates how an initially longitudinal B field (left) acquires an azimuthal component due to rotation (right). In the scenario we are considering in this review, the longitudinal $\mathbf{B}$-field component comes about due to the jet outflow. The result is a helical jet $\mathbf{B}$ field.

the rotation measure (RM), and a linear " $\lambda^{2}$ " dependence for the polarisation angles observed at three or more wavelengths immediately suggests the action of Faraday rotation.

Thus, the observed plane of polarisation will depend on (i) the geometry of the $\mathbf{B}$ field giving rise to the synchrotron radiation, (ii) the optical depth of the emitting region and (iii) the amount of Faraday rotation present.

This review will consider various recent observational results in the context of the hypothesis that many AGN jets have helical magnetic fields. The number of AGN for which parsec-scale polarisation information is available has recently increased considerably with the publication of the first-epoch linear polarisation (Lister \& Homan 2005) and circular polarisation (Homan \& Lister 2006) results for the MOJAVE sample, which contains 133 radio-bright AGN that are being monitored regularly with the VLBA at $15 \mathrm{GHz}$; results of the MOJAVE observations will be considered in various contexts throughout the review.

\section{Why might many AGN jets have helical B fields?}

From a theoretical point of view, it would be very natural if many, or possibly even all, AGN jets are associated with helical B fields, produced essentially by the "winding up" of an initial "seed" field threading the accretion disc by the combination of the rotation of the central black hole and accretion disc and the jet outflow (e.g. Nakamura, Uchida, \& Hirose 2001; Meier, Koide \& Uchida 2001, e.g., Fig. 1 above; Lovelace et al. 2002; Hujeirat et al. 2003; Lynden-Bell 2003; Tsinganos \& Bogovalov 2002). The possibility that toroidal field components develop in association with currents flowing in the jet has also been considered (Pariev, Istomin \& Bereznyak 2003; Lyutikov 2003). Indeed, these two ideas are not unrelated: whether or not it is related to a helical $\mathbf{B}$ field, if there is a dominant toroidal $\mathbf{B}$-field component, basic physics tells us that there must be currents flowing in the region enclosed by the field.

This very plausible theoretical picture provides strong motivation to search for evidence of helical/toroidal B-field structures associated with the relativistic jets of AGN. The remainder of 
this section will consider various observational evidence - some of it compelling - that, indeed, many AGN jets do have helical B fields. Before turning to this evidence, we will first consider the question of the angle at which the jet of a typical radio-loud AGN is viewed in the observer and source frames.

\subsection{Viewing angle in the observer and source frames}

Suppose a particle moves with velocity $\mathbf{u}^{\prime}$ in a frame that moves with velocity $\mathbf{v}$ relative to the observer's (unprimed) frame. The observed components of the particle's velocity parallel and perpendicular to $\mathbf{v}$ will be (Rybicki \& Lightman 1979)

$$
\begin{gathered}
u_{\|}=\frac{u_{\|}^{\prime}+v}{\left(1+v u_{\|}^{\prime} / c^{2}\right)} \\
u_{\perp}=\frac{u_{\perp}^{\prime}}{\gamma\left(1+v u_{\|}^{\prime} / c^{2}\right)}
\end{gathered}
$$

where $\gamma=1 / \sqrt{1-v^{2} / c^{2}}$ is the Lorentz factor for the motion of the source. The angles $\theta^{\prime}$ and $\theta$ along which the particle is observed to move in the two frames are related by the expression:

$$
\begin{aligned}
\tan \theta & =\frac{u_{\perp}}{u_{\|}}=\frac{u_{\perp}^{\prime}}{\gamma\left(u_{\|}^{\prime}+v\right)} \\
& =\frac{u^{\prime} \sin \theta^{\prime}}{\gamma\left(u^{\prime} \cos \theta^{\prime}+v\right)}
\end{aligned}
$$

If the moving "particle" is a photon, so that $u^{\prime}=c$, this reduces to

$$
\begin{aligned}
\tan \theta & =\frac{\sin \theta^{\prime}}{\gamma\left(\cos \theta^{\prime}+\beta\right)} \\
\cos \theta & =\frac{\cos \theta^{\prime}+\beta}{1+\beta \cos \theta^{\prime}}
\end{aligned}
$$

where $\beta=v / c$. Thus, a photon emitted at right angles to $\mathbf{v}$ in the source rest frame $\left(\theta^{\prime}=\pi / 2\right)$ will be observed to propagate at the angle

$$
\begin{aligned}
\cos \theta & =\beta \\
\sin \theta & =\frac{1}{\gamma}
\end{aligned}
$$

When $\gamma$ is large, $\sin \theta$ will be small, and

$$
\theta \simeq \frac{1}{\gamma}
$$


This is the phenomenon that leads to the well-known "beaming" of the source radiation in the forward direction of its motion, but it also has another observational consequence that is less often considered: if we are observing an AGN jet at a relatively small angle to the line of sight, we are detecting synchrotron photons that were emitted at relatively large angles to the jet axis in the source frame. More precisely, if we are viewing the jet at an angle $\theta \approx 1 / \gamma$, the photons we detect were emitted at roughly $90^{\circ}$ to the jet axis in the source rest frame. This will have interesting observational consequences, as will be discussed below.

\section{Observational evidence for AGN jets with helical B fields}

\subsection{Extended regions of transverse B field}

In general, a helical $\mathbf{B}$ field can be described as a superposition of a toroidal and a longitudinal component. The larger the pitch angle (angle between the helix axis (jet axis) and $\mathbf{B}$ vector), i.e. the more tightly wound the helix, the stronger the toroidal relative to the longitudinal component. If we are viewing a jet with a helical $\mathbf{B}$ field with a pitch angle that is fairly high at an angle that is not too close to the jet axis in the source (jet) rest frame, the overall jet $\mathbf{B}$ field will be dominated by the toroidal component - this will be manifest as an observed jet $\mathbf{B}$ field that is orthogonal to the jet direction.

Indeed, it is not at all uncommon for the jets of core-dominated AGN to display regions in which the predominant inferred $\mathbf{B}$ field is orthogonal to the jet direction; BL Lac objects, in particular, show a distinct tendency for their VLBI jets to have such B fields (e.g. Gabuzda, Pushkarev \& Cawthorne 2000 and references therein, Lister \& Homan 2005). These fields were initially interpreted as being associated with transverse shocks that enhance the $\mathbf{B}$-field component in the plane of compression (Laing 1980; Hughes, Aller \& Aller 1989; Gabuzda et al. 1989). Indeed, it is very likely that some individual strongly polarised VLBI components whose $\mathbf{B}$ fields are perpendicular to the direction of propagation do represent shocked regions.

However, a number of AGN jets have been found to display extended, at times not particularly compact, regions with $\mathbf{B}$ fields orthogonal to the jet direction; an example is shown in Fig. 2. In some cases, the jet $\mathbf{B}$ fields remain close to orthogonal to the jet direction even in the case of very substantial bending (e.g. 0954+658: Gabuzda \& Cawthorne 1996, 1803+784: Gabuzda 1999, 1749+701: Lyutikov, Pariev \& Gabuzda 2005). It is possible to interpret this behaviour as reflecting the presence of a whole series of relativistic shocks along the jet, each enhancing the local transverse $\mathbf{B}$ field, but this picture is clearly somewhat contrived. In contrast, in the alternative view that the observed transverse $\mathbf{B}$ fields are associated with the dominant toroidal component of an intrinsic jet $\mathbf{B}$ field, maintenance of the orthogonal field orientation as the jets curve is natural. For this reason, the presence of extended regions in AGN jets with $\mathbf{B}$ fields orthogonal to the jet direction represents strong circumstantial evidence supporting the hypothesis that these jets are associated with helical $\mathbf{B}$ fields.

\subsection{Spine+Sheath jet $B$-field structures}

Attridge, Roberts \& Wardle (1999) reported the presence of a central "spine" of orthogonal B field with a "sheath" of longitudinal B field at the edge of the VLBI jet of 1055+018 (Fig. 3, 


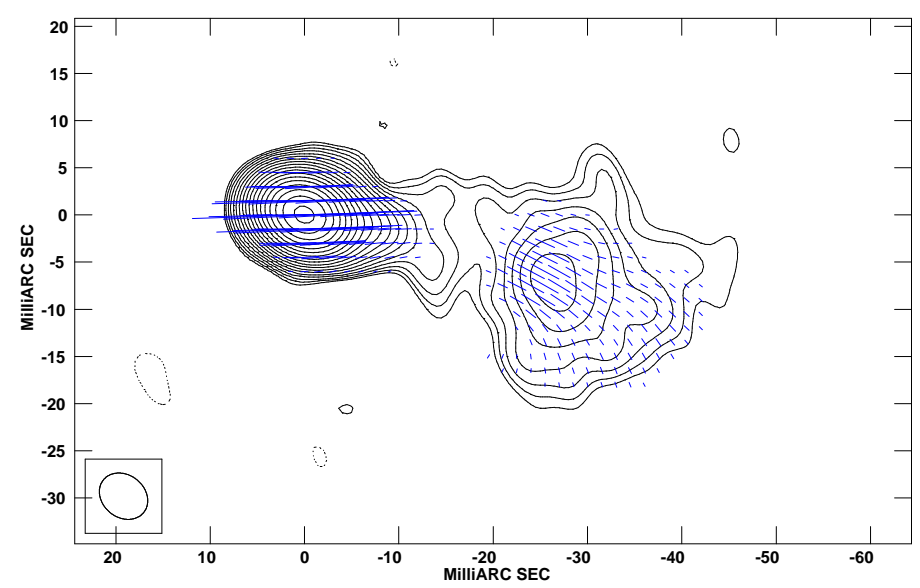

Figure 2: $18-\mathrm{cm}$ total intensity contours of the BL Lac object $1803+784$ with polarisation $\mathrm{E}$ vectors (corrected for the integrated rotation measure of $-61 \mathrm{rad} / \mathrm{m}^{2}$; Wrobel 1993, Pushkarev 2001) superposed. The polarisation vectors are well aligned with the jet direction throughout the jet (see Gabuzda \& Chernetskii 2003).

left). They interpreted this structure as being a manifestation of the combination of a central region whose $\mathbf{B}$ field was determined by shock compression, with an interaction with the surrounding medium giving rise to regions of longitudinal field at the edges of the jet due to shear. While this may be a reasonable interpretation if this type of structure is present in a relatively few individual sources, it is now becoming clear that such "spine+sheath" B-field structures are fairly common. Multi-frequency VLBI images of three more AGN displaying similar polarisation structures are presented by Pushkarev et al. (2005), and numerous other examples can be found among the sources monitored by the MOJAVE project (Lister \& Homan 2005); an example is shown in Fig. 3 . In this light, the interpretation suggested by Attridge et al. (1999) begins to sound implausible as a general explanation for this phenomenon, since it requires the action of two independent factors: the formation of transverse relativistic shocks all along the VLBI jet (see also the arguments about this in the previous section) and the presence of shear with the surrounding medium, which enhances the longitudinal $\mathbf{B}$ field at the jet edges.

In fact, $\mathbf{B}$-field structures with a central region of predominantly transverse $\mathbf{B}$ field and edges with predominantly longitudinal $\mathbf{B}$ field relative to the jet direction can come about in a very natural way if the jet has a helical $\mathbf{B}$ field with a fairly high pitch angle (i.e., it is fairly tightly wound), as has been pointed out by Lyutikov et al. (2005); see Fig. $\theta$. This represents a simpler explanation for the observed spine+sheath $\mathbf{B}$-field structures, since it does not require the simultaneous action of two independent factors, and does not require the presence of a whole series of transverse shocks all along the jet. In addition, in the case of a helical $\mathbf{B}$ field, we would expect the degree of polarisation to increase toward the edges of the jet (Lyutikov et al. 2005), as has been reported for some sources (Pushkarev et al. 2005, Zavala \& Taylor 2005).

Thus, helical jet $\mathbf{B}$ fields offer a simple explanation for the spine+sheath $\mathbf{B}$-field structures that are fairly commonly observed among compact AGN. Based on the principle of Occam's Razor, which gives preference to the simplest of various possible interpretations of observed phenomena, it seems reasonable to take helical jet $\mathbf{B}$ fields as the most likely explanation for these structures, in 

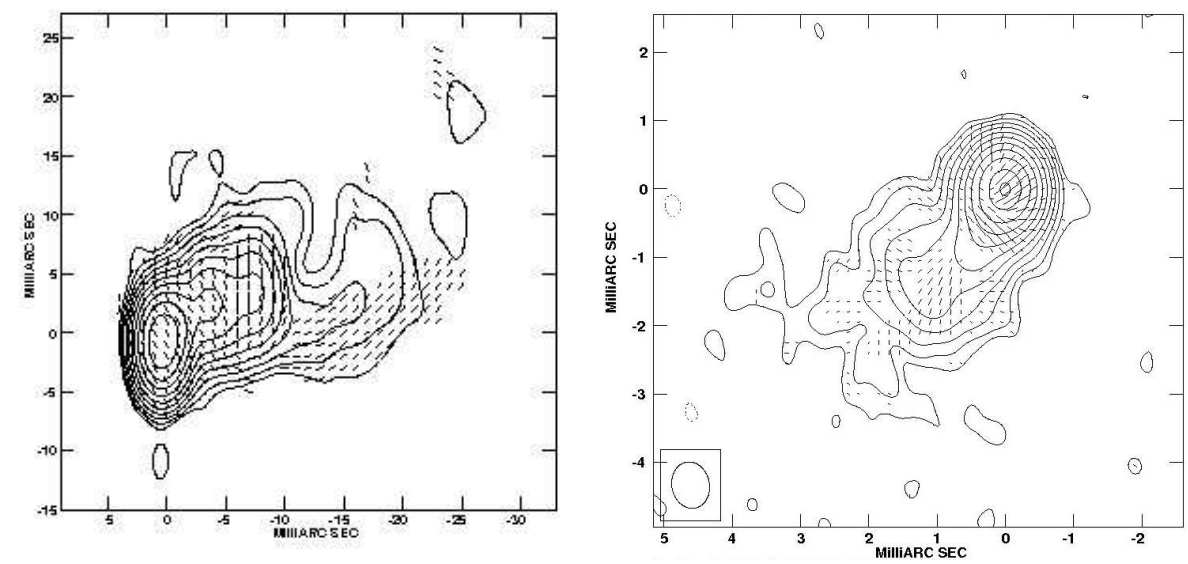

Figure 3: Two examples of "spine+sheath" polarisation structures. Left: the first example discovered, 1055+018, taken from Attridge et al. (1999). Shown are 6-cm total intensity contours with pseudo-B vectors (polarisation $\mathbf{E}$ vectors rotated by $90^{\circ}$ ) superposed. Right: the BL Lac object 0300+470 (Gabuzda, O'Dowd $\&$ Aller, in preparation). Shown are 2 -cm total intensity contours with the observed polarisation $\mathbf{E}$ vectors superposed.

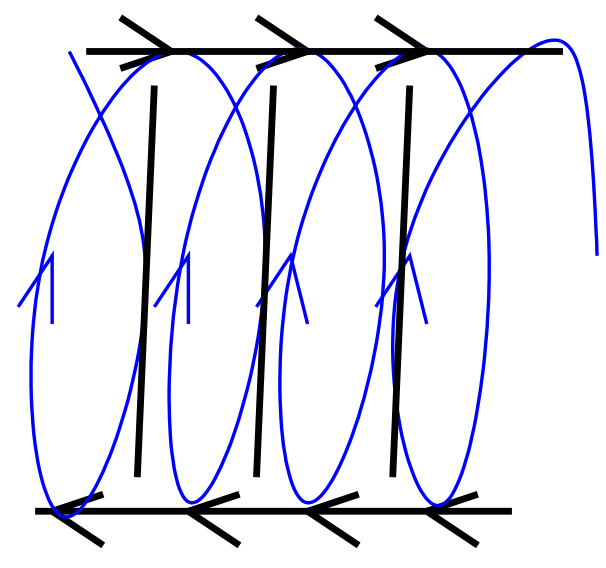

Figure 4: Schematic illustration of how a helical jet $\mathbf{B}$-field geometry can give rise to a central region of $\mathbf{B}$ field orthogonal to the jet with regions of longitudinal $\mathbf{B}$ field near the edges of the jet.

the absence of other observational evidence supporting the hypothesis that the longitudinal $\mathbf{B}$ fields near the edges of the jet are associated with an interaction with the surrounding medium. We will consider this question further below.

\subsection{Transverse Faraday-rotation gradients}

Although the presence of extended regions in the jet where the $\mathbf{B}$ field is orthogonal to the jet direction and of B-field structures with a central "spine" of orthogonal field and a "sheath" of longitudinal field can provide strong circumstantial evidence that the corresponding jets have helical B fields, there is another phenomenon that represents a real "smoking gun" in terms of revealing the presence of helical jet $\mathbf{B}$ fields: the presence of a systematic gradient in the Faraday rotation measure across the jet. The origin of this Faraday-rotation gradient is the systematic variation in 


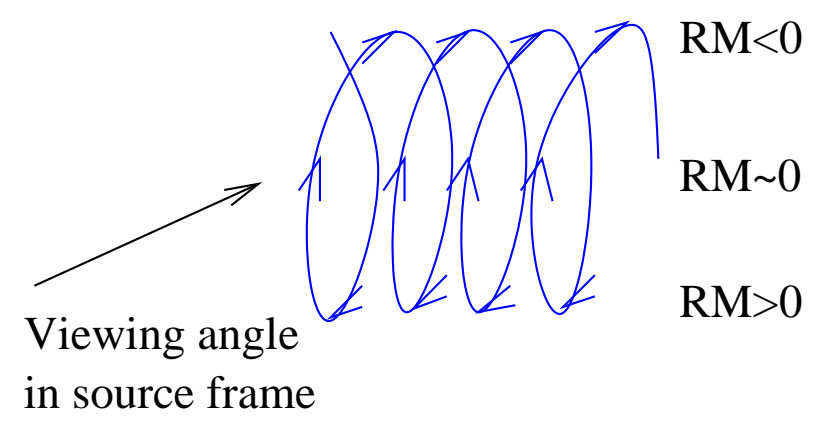

Figure 5: Expected behaviour of the observed Faraday-rotation gradient transverse to a jet with a helical B field for the case when the jet is viewed at approximately $90^{\circ}$ to the jet (helix) axis in the source frame. The rotation measure has different signs on either side of the jet, due to the different directions of the line-of-sight component of the helicalB field.

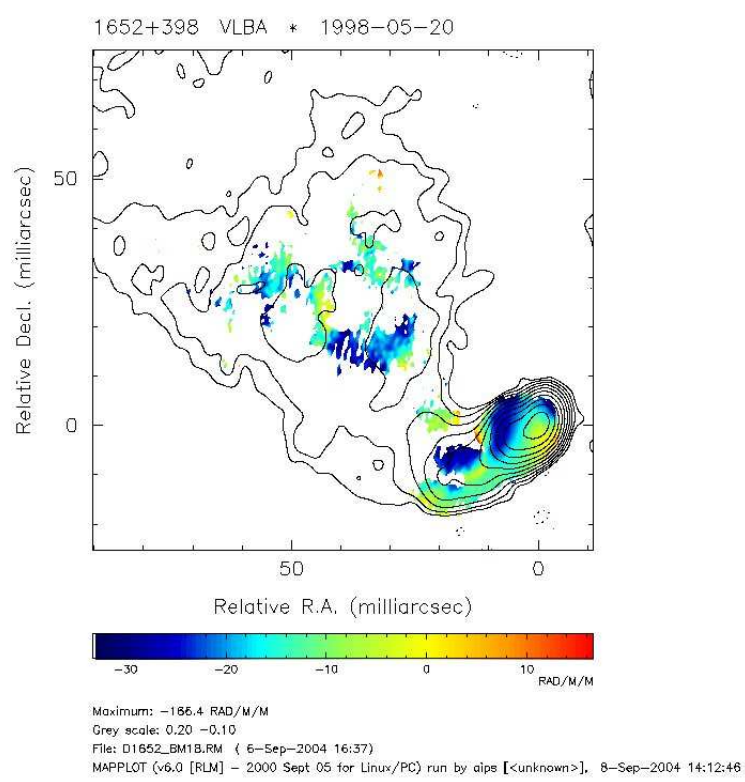

Figure 6: Faraday-rotation gradient transverse to the jet in 1652+398 (Mrk501; Croke \& Gabuzda, in preparation). Shown are $18-\mathrm{cm}$ total intensity contours with the rotation-measure distribution derived from simultaneous $3.6 \mathrm{~cm}+6 \mathrm{~cm}+13 \mathrm{~cm}+18 \mathrm{~cm}$ VLBA polarisation data superposed. The transverse RM gradient is present over a very extended region of the observed parsec-scale jet.

the line-of-sight component of the helical $\mathbf{B}$ field across the jet; recall that it is the line-of-sight component of the $\mathbf{B}$ field in the region of Faraday rotation that appears in the expression for the the observed polarisation angle above. This effect had, in fact, been predicted earlier by Blandford (1993). As an example, if the helical $\mathbf{B}$ field is viewed at an angle that is roughly $90^{\circ}$ to the jet/helix axis in the source frame (Fig. 5), we would expect the rotation measure RM to be positive at one edge, roughly zero in the central region of the jet and negative at the other edge. As the viewing angle in the source frame moves away from $90^{\circ}$ to the jet/helix axis, the observed RM gradient becomes more and more asymmetric, encompassing RM values of only one sign in some cases.

Rotation-measure gradients transverse to the jet direction, interpreted as evidence that these 
jets have helical $\mathbf{B}$ fields, have now been reported for nearly a dozen AGN (3C273: Asada et al. 2002; Zavala \& Taylor 2005; Chen 2005; several BL Lac objects: Gabuzda, Murray \& Cronin 2004; Mahmud \& Gabuzda, these proceedings). Note that Gabuzda et al. (2004) and Mahmud \& Gabuzda (these proceedings) first subtracted the effect of the known integrated rotation measures, taken to occur in our Galaxy, so that the RM distributions they present correspond to Faraday rotation occurring in the immediate vicinity of the AGN. The detection of these transverse RM gradients represents the strongest evidence available that helical $\mathbf{B}$ fields are associated with these - and possibly many other - AGN jets. One example is shown in Fig. 6 .

Of course, it is also possible to imagine situations when a gradient in the Faraday rotation roughly across a VLBI jet occurs, not due to a systematic variation in the line-of-sight $\mathbf{B}$-field component, but due to a gradient in the density of thermal electrons in the region surrounding the jet. This could be the case, for example, if the jet were propagating through a non-uniform medium that was denser on one side of the jet than on the other. In this regard, it is important to point out a crucial discriminator: while transverse RM gradients with RM values of a single sign that increase toward one edge of the jet could potentially be caused by either a helical jet $\mathbf{B}$ field or a density gradient in the surrounding medium, transverse RM gradients that display one sign at one edge of the jet, pass through zero and then display the other sign at the other edge of the jet are a clear and unambiguous sign of a helical jet $\mathbf{B}$ field. It is therefore highly significant that transverse RM gradients displaying both signs have been observed for $0735+178,0745+241$, $1652+398,1803+784$ and $2155-152$, and possibly $3 \mathrm{C} 273$ and $1418+546$; of the ten cases reported thus far, only three $(0256+075,0820+225,3 \mathrm{C} 371)$ show RM gradients that clearly display only one sign down to the minimum detected RM. Note that, in all cases except for 3C273, whose integrated RM is negligible (Rusk 1988), the effect of the integrated RM has been subtracted out, so that the RM distribution we are considering arises in the immediate vicinity of the AGN. Thus, in the majority of cases, the only plausible explanation for the observed transverse RM gradients is that the corresponding jets have helical $\mathbf{B}$ fields.

As Sikora et al. (2005) have pointed out in connection with the results of Gabuzda et al. (2004), the lack of deviations from a $\lambda^{2}$ wavelength dependence for the observed polarisation angles indicates that the Faraday rotation must be external, suggesting that the associated helical $\mathbf{B}$ field may surround the jet, but not necessarily fill the jet volume; alternatively, it may be that the helical field fills the jet volume, but the thermal electrons required for the Faraday rotation do not.

\subsection{Clues from circular polarisation measurements}

One of the most exciting recent developments in VLBI studies of AGN is the detection of small but significant amounts of circular polarisation (CP) on milli-arcsecond scales (e.g. Homan, Attridge \& Wardle 2001). The amount of VLBI-scale CP information available has recently been very considerably increased with the publication of first-epoch $\mathrm{CP}$ measurements for all the sources in the MOJAVE sample (Homan \& Lister 2006). Various possible mechanisms for the generation of CP in relativistic astrophysical jets are discussed, for example, by Macquart \& Melrose (2000), Wardle \& Homan $(2001,2003)$ and Beckert \& Falcke (2002). Synchrotron radiation includes a very small amount of intrinsic CP (Legg \& Westfold 1968), but the degrees of CP that are observed on VLBI scales (typically a few tenths of a percent, but reaching more than one percent in some cases) are high enough to make this mechanism implausible. It is generally agreed that a much 
more plausible mechanism is the Faraday conversion of linear-to-circular polarisation during the propagation of a linearly polarised electromagnetic wave through a magnetized plasma (Jones \& O’Dell 1977; Jones 1988).

In order for Faraday conversion to operate, the magnetic field in the conversion region $\mathbf{B}_{\text {conv }}$ must have a non-zero component parallel to the plane of linear polarisation. For this reason, as has been pointed out by Wardle \& Homan (2001), a helical B field provides an interesting example of an overall ordered $\mathbf{B}$ field geometry that can potentially facilitate linear-to-circular conversion: synchrotron radiation emitted at the "far" side of the helical field relative to the observer can undergo conversion in the "near" side of the helical field.

This raises the question of whether the CP detected in AGN on VLBI scales could be generated in helical $\mathbf{B}$ fields associated with the corresponding AGN jets. Inspection of the MOJAVE sources in which CP was detected (Homan \& Lister 2006) reveals that a number of them show various properties that can be associated with the presence of helical jet B fields, such as extended regions of transverse $\mathbf{B}$ field, spine+sheath jet $\mathbf{B}$-field structures and transverse RM gradients, providing indirect evidence that at least some of the detected CP may be associated with helical jet $\mathbf{B}$ fields. It is also intriguing that extended regions of CP clearly in the VLBI jet, well away from the optically thick core region, have now been detected in several cases (Homan \& Lister 2006, Vitrishchak \& Gabuzda 2007) - linear to circular conversion in helical B fields associated with these jets could explain this result in a natural way.

\subsection{Connection with superluminal speeds}

The jets of virtually all compact, flat-spectrum, radio-loud AGN display superluminal motions when images obtained at several different observing epochs are compared. This is consistent with the fact that we believe we are viewing these jets at relatively small angles to the jet axes in our own rest frame; the observed apparent speed $\beta_{a p p}=v_{a p p} / c$ for the case of a jet component that is moving with an intrinsic speed $\beta=v / c$ at an angle $\theta$ to the line of sight is given by the well known relation

$$
\beta_{a p p}=\frac{\beta \sin \theta}{1-\beta \cos \theta}
$$

Although there is considerable overlap between the two distributions, it has long been known that different types of AGN display somewhat different typical superluminal speeds, with, on average, lower apparent speeds being observed in BL Lac objects than in quasars (Gabuzda et al. 1994, 2000; Kellermann et al. 2004). In principle, this systematic difference could be associated with a difference in either the intrinsic flow speeds in the jet or the angles made by the jets to the line of sight. If due primarily to a difference in the characteristic angles of the jets to the line of sight, the lower speeds observed in BL Lac objects would indicate that their jets are typically viewed at angles $\theta$ that are further from the angle $1 / \Gamma$ that maximises the observed apparent speed than is the case for quasars, where $\Gamma$ is the bulk Lorentz factor of the jet. BL Lac objects would be observed to have relatively low superluminal speeds if $\theta$ were either appreciably larger or appreciably smaller than $1 / \Gamma$. The strong variability of BL Lac objects argues against the idea that their jets are typically viewed at relatively large angles to the line of sight. Further, Gabuzda et al. (2000) pointed out that the lack of evidence that BL Lac objects are typically more beamed than quasars argues 
against a scenario in which the jets of BL Lac objects are viewed at angles appreciably smaller than the viewing angles of quasar jets. Accordingly, Gabuzda et al. (2000) conclude that the lower apparent superluminal speeds of BL Lac objects most likely reflect lower average bulk Lorentz factors for their jets.

It has independently been suggested by Gabuzda et al. (2000) and Asada et al. (2002) that the observed difference in the typical superluminal speeds of BL Lac objects and quasars could be related to another systematic difference in the VLBI properties of these two types of AGN: the VLBI jets of BL Lac objects/quasars most often display predominantly $\mathbf{B}$ fields that are orthogonal to/aligned with the jet direction. Let us suppose that both quasars and BL Lac objects characteristically have helical jet $\mathbf{B}$ fields that come about due to the combination of rotation of the central black hole/accretion disc and the jet outflow. If the intrinsic outflow speeds of quasars are, on average, higher than those of BL Lac objects, this might mean that the ratios of their outflow speeds to their central rotational speeds are also higher, giving rise to helical jet $\mathbf{B}$ fields that are less tightly wound. On the other hand, lower outflow speeds in BL Lac objects might lead to the helical $\mathbf{B}$ fields associated with their jets being more tightly wound. Thus, it may be possible to understand the observed systematic differences in the VLBI properties of BL Lac objects and quasars within a single scenario in which a tendency for lower outflow speeds in BL Lac objects compared to quasars leads to a tendency for more tightly wound helical jet $\mathbf{B}$ fields in BL Lac objects, which are manifest as a predominance for jet $\mathbf{B}$ fields orthogonal to the jet direction in these sources.

\subsection{Unexpected results from high-frequency core Faraday-rotation measurements}

Virtually all VLBI Faraday-rotation studies have been carried out at various combinations of wavelengths in the range from $2 \mathrm{~cm}$ to $6 \mathrm{~cm}$, based on the expectation that this would provide a reasonable compromise between resolution and sensitivity (Taylor 1998, 2000; Reynolds, Cawthorne \& Gabuzda 2001; Gabuzda \& Chernetskii 2003; Zavala \& Taylor 2002, 2003, 2004). These studies indicated typical core rotation measures in the rest frame of the AGN of $|R M| \simeq 500-5000$ for quasars and $|R M| \simeq 200-500$ for BL Lac objects.

Gabuzda et al. (2006) have recently reported RM maps based on VLBA polarisation data at $7 \mathrm{~mm}, 1.3 \mathrm{~cm}$ and $2 \mathrm{~cm}$, obtained nearly simultaneously with optical polarisation measurements. Unexpectedly, these indicate core RMs that are typically a factor of a few to more than an order of magnitude higher than those deduced for these same objects from $2 \mathrm{~cm}-6 \mathrm{~cm}$ VLBA polarisation data, up to $R M \simeq 6000 \mathrm{rad} / \mathrm{m}^{2}$ for BL Lac; Mutel, Denn \& Dreier (2005) have reported similarly high core RMs for BL Lac based on $7 \mathrm{~mm}-2 \mathrm{~cm}$ VLBA polarisation data. It seems reasonable to interpret this as revealing the presence of higher densities of thermal gas on the smaller scales probed by the higher-frequency observations.

Intriguingly, in several cases, the sign of the core RM was different in the $7 \mathrm{~mm}-2 \mathrm{~cm}$ and $2 \mathrm{~cm}-6 \mathrm{~cm}$ measurements (O'Sullivan \& Gabuzda, these proceedings). As we saw above, this must correspond to a change in the orientation of the line-of-sight component of the $\mathbf{B}$ field in the region giving rise to the Faraday rotation. O'Sullivan \& Gabuzda (these proceedings) suggest that these changes in the line-of-sight $\mathbf{B}$ field may be associated with bends in the jet, and suggest several scenarios in which changes in the orientation of the line-of-sight $\mathbf{B}$ field component could come about in the case of modest bends of a VLBI jet with a helical $\mathbf{B}$ field. 
In fact, it is also not at all uncommon for different regions in the VLBI structure of a given source (core and jet, different components in the jet) at a given epoch to have RMs with different signs; numerous examples can be found, for example, in Zavala \& Taylor (2003, 2004). If this is true for the Faraday rotation occurring in the immediate vicinity of the AGN, then this provides direct information about changes in the orientation of the line-of-sight component of the $\mathbf{B}$ field in the region giving rise to the Faraday rotation. Indeed, different RM signs in different regions in the VLBI structure have been observed for sources whose integrated RMs (presumed to occur predominantly in our Galaxy) are known and can be subtracted off, so that we can know that the distribution of Faraday rotation occurring in the immediate vicinity of the AGN displays different signs in different components of the VLBI structure.

In both the scenarios considered above (different core RM signs for VLBI data obtained in different frequency ranges, different RM signs for different components in the VLBI structure), the RM sign provides information about the $\mathbf{B}$-field structure perpendicular to the plane of the sky, while the polarisation angles provide information about the $\mathbf{B}$-field structure projected onto the plane of the sky. This provides hope that studies of changes in the sign of the observed RM analysed jointly with other VLBI properties, such as apparent bends, changes in apparent superluminal speeds, or the presence and characteristics of transverse RM gradients, can be used to at least partially reconstruct the three-dimensional structure of the jets.

\section{Directions for future work}

The above sections summarize the available observational evidence that the jets of at least some compact radio-loud AGN have helical B fields (see also Lyutikov et al. 2005). Such evidence includes the presence of extended regions in VLBI jets with $\mathbf{B}$ field orthogonal to the jet direction, stretches of VLBI jets where the inferred $\mathbf{B}$ field remains nearly orthogonal to the jet direction even in the case of substantial bending of the jet (i.e., the observed polarisation E vectors "follow" the VLBI jet) and the common occurrence of spine+sheath jet polarisation structures. However, the most convincing evidence for the presence of helical jet $\mathbf{B}$ fields in compact, radio-loud AGN is provided by the detection in the last few years of Faraday-rotation gradients across the VLBI jets (i.e., transverse to the VLBI jet direction) of about ten sources; here, the systematic rotation-measure gradient is interpreted as being due to the systematic change in the line-of-sight component of the helical jet B field. Finally, a helical jet B-field geometry can facilitate the Faraday conversion of linear to circular polarisation, and it may be that the non-negligible degrees of circular polarisation detected in the VLBI cores, and in some cases also VLBI jets, of a substantial fraction of compact, flat-spectrum, radio-loud AGN come about because the jets of these sources have helical $\mathbf{B}$ fields. In particular, this could explain the tendency for the sign of the observed circular polarisation to remain constant over many years, as well as the recent detection of circular polarisation in extensive regions of the VLBI jets of several sources, well away from the optically thick core region.

Unfortunately, it is often not easy to interpret these observational results unambiguously: jet regions with Borthogonal to the jet direction could represent either the toroidal component of an intrinsic jet $\mathbf{B}$ field or regions of shock compression; spine+sheath polarisation structures arise naturally in the case of helical jet $\mathbf{B}$ fields, but could in principle also come about due to the combination of a shocked central region of the jet and shear interaction between the edges of the 
jet and the surrounding medium. While transverse RM gradients are certainly expected in the case of a helical jet $\mathbf{B}$ field, they could also come about due to inhomogeneity in the thermal-electron distribution in the plasma surrounding the jet.

One of the key challenges facing us is thus to find robust observational ways to distinguish between these various interpretations. In this regard, the case for a helical jet $\mathbf{B}$ field becomes stronger if more than one manifestation of such fields are observed simultaneously - for example, extended regions of orthogonal jet $\mathbf{B}$ field observed together with a systematic transverse gradient in the observed Faraday rotation measure, a spine+sheath polarisation structure observed together with a transverse RM gradient, or a transverse RM gradient observed together with a detection of circular polarisation in the jet. It may be difficult formally to "prove" that these combinations of phenomena are associated with the presence of helical jet $\mathbf{B}$ fields rather than the combined action of two or more factors - shock compression plus inhomogeneity in the surrounding medium, shock compression plus interaction with the surrounding medium, shock compression plus Faraday conversion in plasma surrounding the jet - but the principle of Occam's Razor dictates that the helical jet B-field interpretation should at least tentatively be given preference, since it is a single factor that can account for multiple observed phenomena.

One case that seems quite unambiguous is when a transverse RM gradient is present and displays different signs on opposite sides of the jet. The different RM signs indicate different directions for the line-of-sight $\mathbf{B}$ field giving rise to the Faraday rotation, and the presence of RMs with different signs on different sides of the jet is natural in the case of a helical jet $\mathbf{B}$ field (at least for some combinations of viewing angle and helical pitch angle), but is difficult to explain otherwise. It is therefore important to note that, of the ten sources for which transverse jet RM gradients have been reported thus far, only three have RM gradients that clearly display only one sign down to the minimum detected RM. Thus, for most of the observed transverse RM gradients, the only reasonable interpretation is that the RM gradient is associated with a helical jet $\mathbf{B}$ field.

Further, we can ask how we can observationally distinguish transverse RM gradients displaying a single sign due to helical jet $\mathbf{B}$ fields or non-uniform surrounding media. The question here is essentially, is the observed RM gradient due primarily to a gradient in the line-of-sight component of the $\mathbf{B}$ field or a gradient in the thermal-plasma density? One possible test may be how close to orthogonal the RM gradient is to the jet direction, although this may not be easy to determine in the case of a somewhat bent jet. Another possibility is to search for further evidence of a higher concentration of thermal plasma on the side of the jet with the higher rotation measure, such as depolarisation or low-frequency absorption; finding such evidence would lend weight to the hypothesis that the observed RM gradient was associated with a non-uniform surrounding medium. Numerical computations of the total intensity, polarisation and RM profiles across the jet expected for the case of a helical jet B field (e.g. Uchida et al. 2004, Lyutikov et al. 2005, Papageorgiou 2006) and comparison with the observed profiles could also be useful in this regard.

It is also of interest to try to find ways to observationally distinguish a spine+sheath polarisation structure due to the joint action of transverse shocks+shear or to a helical jet $\mathbf{B}$ field. Again, comparison of the observed total intensity and polarisation profiles across the jet with computed profiles for a helical jet B field could be useful for this purpose (e.g. Uchida et al. 2004, Lyutikov et al. 2005, Papageorgiou 2006). For example, a systematic and monotonic increase in the degree of polarisation toward the edges of the jet is expected for a spine+sheath polarisation structure caused 
by a helical jet $\mathbf{B}$ field, while, in general, one would probably not expect such systematic behaviour in the case of shocks+shear (since they represent a superposition of two independent phenomena). In fact, a systematic increase in the degree of polarisation toward the edges of the jet has been observed in at least two sources that display clear evidence for helical jet B fields $-1652+398$ (Mrk501; Pushkarev et al. 2005) and 3C273 (Zavala \& Taylor 2005).

Finally, in cases when we can be reasonably confident that a transverse jet $\mathbf{B}$ field represents the predominant component of a $\mathbf{B}$ field organically related to the jet itself, it is of interest to place limits on the longitudinal component of this intrinsic $\mathbf{B}$ field - in other words, is the intrinsic jet $\mathbf{B}$ field purely toroidal or is it helical? One possible diagnostic here is the presence of circular polarisation in the VLBI jet - the generation of circular polarisation via Faraday conversion will be facilitated by a helical jet $\mathbf{B}$ field, but not a purely toroidal field. The implications of the detection of circular polarisation in the VLBI core are less clear-cut, but the detection of core circular polarisation nevertheless provides some evidence supporting the idea that the jet $\mathbf{B}$ field is not purely toroidal, based on the hypothesis that the observed "core" circular polarisation is actually generated in the innermost VLBI jet, on scales that are larger than the intrinsic core but smaller than the resolution provided by the VLBI array used.

There are a growing number of sources for which reasonably unambiguous evidence for helical jet $\mathbf{B}$ fields is available - those displaying transverse RM gradients with opposite signs on opposite sides of the jet, and those displaying multiple "symptoms" of helical fields. This compels us to take very seriously the idea that some, possibly many, AGN jets have helical B fields. This is not surprising, since such fields come about very naturally as a consequence of the joint action of rotation of the central black hole+accretion disc and the jet outflow. The continued accumulation of firm observational evidence for AGN jets with helical B fields will increasingly validate this theoretical picture, and demonstrate that we must look at these jets as fundamentally electromagnetic, current-carrying structures if we wish to fully understand their nature.

\section{References}

[1] Asada K. et al. 2002, PASJ, 54, L39

[2] Beckert T. \& Falcke H. 2002, A\&A, 388, 1106

[3] Blandford R. D. 1993, in Astrophysical Jets (Cambridge University Press), p. 26

[4] Burn B. J. 1966, MNRAS, 133, 67

[5] Chen T. 2005, PhD Thesis, Brandeis University, Brandeis MA, USA

[6] Gabuzda D. C. 1999, New Astronomy Reviews, 32, 691

[7] Gabuzda D. C. \& Cawthorne T. V. 1996, MNRAS, 283, 759

[8] Gabuzda D. C., Cawthorne T. V., Roberts D. H. \& Wardle J. F. C. 1989, ApJ, 347, 701

[9] Gabuzda D. C. \& Chernetskii V. A. 2003, MNRAS, 339, 669

[10] Gabuzda D. C. \& Gómez J. L. 2001, MNRAS, 320, 49

[11] Gabuzda D. C., Mullan C. M., Cawthorne T. V., Wardle J. F. C. \& Roberts D. H. 1994, ApJ, 435, 140

[12] Gabuzda D. C., Murray E., Cronin P. 2004, MNRAS, 351, L89 
[13] Gabuzda D. C., Pushkarev A. B. \& Cawthorne T.V. 2000, MNRAS, 319, 1109

[14] Gabuzda D. C., Rastorgueva E. A., Smith P. S. \& O'Sullivan S. P. 2006, MNRAS, 369, 1596

[15] Homan D. C., Attridge J. M. \& Wardle J. F. C. 2001, ApJ, 556, 113 319, 1109

[16] Homan D. C. \& Lister M. L. 2006, AJ, 131, 1262

[17] Hughes P. A., Aller H. D. \& Aller M. A. 1989, ApJ, 341, 68

[18] Hujeirat A., Livio M., Camenzind M. \& Burkert A. 2003, A\&A, 408, 415

[19] Jones T. W. 1988, ApJ, 332, 678

[20] Jones T. W. \& O’Dell S.L. 1977, ApJ, 214, 522

[21] Kellermann K. I. et al. 2004, ApJ, 609, 539

[22] Laing R. 1980, MNRAS, 193, 439

[23] Legg M. P. C. \& Westfold K. C. 1968, ApJ, 154, 499

[24] Lister M. L. \& Homan D. C. 2005, AJ, 130, 1389

[25] Lovelace R. V. E., Li H., Koldoba A. V., Ustyugova G. V. \& Romanova M. M. 2002, ApJ, 572, 445

[26] Lynden-Bell D. 2003, MNRAS, 341, 1360

[27] Lyutikov M. 2003, New Astron. Rev., 47, 513

[28] Lyutikov M., Pariev V. I. \& Gabuzda D. C. 2005, MNRAS, 360, 869

[29] Macquart J.-P. \& Melrose D. B. 2000, ApJ, 545, 798

[30] Meier D. L., Koide S. \& Uchida Yu. 2001, Science, 291, 84

[31] Mutel, R. L., Denn G. R. \& Dreier C. 2005, in Future Directions in High-Resolution Radio Astronony: The 10th Anniversary of the VLBA, J. Romney \& M. Reid Eds., Astron. Soc. Pac. Conf. Ser., 340 , p. 155

[32] Nakamura M., Uchida Y., Hirose S. 2001, New Astron., 6, 61

[33] Pacholczyk A. G. 1970, Radio Astrophysics. Non-thermal Processes in Galactic and Extragalactic Sources (San Francisco, Freeman)

[34] Papageorgiou A. 2006, PhD thesis, University of Central Lancashire

[35] Pariev V. I., Istomin Ya. N. \& Beresnyak A. R. 2003, A\&A, 403, 805

[36] Pushkarev A. B. 2001, Astron. Rep., 45, 667

[37] Pushkarev A. B., Gabuzda D. C., Vetukhnovskaya Yu. N. \& Yakimov V. E. 2005, MNRAS, 356, 859

[38] Reynolds C., Cawthorne T. V. \& Gabuzda D. C. 2001, MNRAS, 327, 1071

[39] Rusk R. E. 1988, PhD Thesis, University of Toronto, Toronto ON, Canada

[40] Rybicki G. B. \& Lightman A. P. 1979, Radiative Processes in Astrophysics (Wiley)

[41] Sikora M., Begelman M. C., Madejski G. M. \& Lasota J.-P. 2005, ApJ, 625, 62

[42] Taylor G. B. 1998, ApJ, 506, 637

[43] Taylor G. B. 2000, ApJ, 533, 95

[44] Tsinganos K. \& Bogovalov S. 2002, MNRAS, 337, 553 
[45] Uchida Yu., Kigure H., Hirose Sh., Nakamura M. \& Cameron R. 2004, ApJ, 600, 88

[46] Vitrishchak V. M. \& Gabuzda D. C. 2007, Astronomy Reports, in press

[47] Wardle J. F. C. \& Homan D. A. 2001, in Particles and Fields in Radio Galaxies, R.A. Laing \& K. Blundell Eds., Astron. Soc. Pac. Conf. Ser., 250, p. 15

[48] Wardle J. F. C. \& Homan D. A. 2003, in Circular Polarisation from Relativistic Jet Sources, Astrophys. Space Sci., 288, 143

[49] Wrobel J. M. 1993, AJ, 106, 444

[50] Zavala R. T. \& Taylor G. B. 2002, ApJ, 566, 9

[51] Zavala R. T. \& Taylor G. B. 2003, ApJ, 589, 126

[52] Zavala R. T. \& Taylor G. B. 2004, ApJ, 612, 749

[53] Zavala R. T. \& Taylor G. B. 2005, ApJ, 626, L73 